\title{
Brain natriuretic peptide and high-sensitivity Troponin at 3 hours post emergency department attendance with unexplained syncope predict 90 day outcome
}

\author{
Matthew James Reed, ${ }^{1,2}$ Hannah Brutin, ${ }^{3}$ Neil R. Grubb, ${ }^{4}$ Christopher C. Lang, ${ }^{4}$ Alasdair J. \\ Gray, ${ }^{1,2}$ Kirsty Simpson, ${ }^{1}$ Allan MacRaild, ${ }^{1}$ Christopher J. Weir ${ }^{5}$
}

${ }^{1}$ Emergency Medicine Research Group Edinburgh (EMERGE), Department of Emergency Medicine, Royal Infirmary of Edinburgh; ${ }^{2}$ Edinburgh Acute Care, Usher Institute of Population Health Sciences and Informatics, College of Medicine and Veterinary Medicine, University of Edinburgh; ${ }^{3}$ College of Medicine and Veterinary Medicine, University of Edinburgh; ${ }^{4}$ Department of Cardiology, Royal Infirmary of Edinburgh; ${ }^{5}$ Edinburgh Clinical Trials Unit and Centre for Population Health Sciences, Usher Institute of Population Health Sciences and Informatics, Edinburgh, UK

\begin{abstract}
Correspondence: Matthew James Reed, Department of Emergency Medicine, Royal Infirmary of Edinburgh, 51 Little France Crescent, Edinburgh, EH16 4SA, UK.

Tel.: 0131.242.1448.

E-mail: matthew.reed@nhslothian.scot.nhs.uk

Key words: Ambulatory electrocardiography monitoring; Cardiac arrhythmias; Syncope; Diagnostic tests.

Acknowledgements: thanks to the medical and nursing staff at the Royal Infirmary of Edinburgh for their support with PATCH-ED, and to the Emergency Medicine Research Group Edinburgh research nurses.

Contributions: MR, NG, AG, CL and KS were responsible for the conception and design of the study, KS and AM were responsible for acquisition of data, HB, MR, AM and CW were involved in data analysis and all authors were involved in drafting the article and revising it critically for important intellectual content.
\end{abstract}

Conflict of interest: the authors declare no potential conflict of interest.

Funding: this study was funded by a minor research award from Chest, Heart and Stroke Scotland. MR was supported by an NHS Research Scotland Career Researcher Clinician award. iRhythm provided the Zio ${ }^{\circledR}$ XT monitors free of charge. Abbott provided machine for cardiac biomarker testing (BNP) and testing consumables free of charge. CJW was supported in this work by NHS Lothian via the Edinburgh Clinical Trials Unit. IRhythm, Abbott and the funder had no involvement in the design, conduct or analysis of the study.

Conference presentation: this work was presented at the European Heart Rhythm Association Congress in March 18-20, 2018.

Ethical statement: the study was granted ethical approval by the South East Scotland Research Ethical Committee (15/SS/0072).

Received for publication: 21 June 2018.

Revision received: 15 July 2018

Accepted for publication: 18 July 2018

This work is licensed under a Creative Commons Attribution 4.0 License (by-nc 4.0).

(C) Copyright M.J. Reed et al., 2018

Licensee PAGEPress, Italy

Emergency Care Journal 2018; 14:7652

doi:10.4081/ecj.2018.7652

\begin{abstract}
To investigate if Brain Natriuretic Peptide (BNP) and highsensitivity troponin I predict significant arrhythmia, symptomatic significant arrhythmia and 90-day death and/or Major Adverse Cardiac Event in Emergency Department (ED) unexplained syncope patients investigated with a 14-day ambulatory electrocardiogram (ECG) recorder. Single-centre, prospective, cohort study. Patients 16 years or over presenting within 6 hours of an episode of unexplained syncope were fitted with an ambulatory ECG recorder (Zio ${ }^{\circledR}$ XT monitor) and had high-sensitivity troponin I and BNP measured on ED admission and 3 hours later. Between $17 / 11 / 2015$ and $13 / 06 / 2017,86$ patients were recruited. 24 had significant arrhythmia, 9 symptomatic significant arrhythmia and 26 patients had death and/or Major adverse cardiac events by 90 days. BNP at admission, three hours and change in BNP and high-sensitivity troponin I at three hours were associated with significant arrhythmia and serious outcome. BNP $>20 \mathrm{pg} / \mathrm{mL}$ and/or high-sensitivity troponin I above the $99 \%$ sex specific centile (M>34ng/L, $\mathrm{F}>16 \mathrm{ng} / \mathrm{L})$ at 3 hours had a sensitivity of $100 \%(21 / 21 ; 95 \% \mathrm{CI}$ $81 \%-100 \%)$ and specificity of $43 \%(16 / 37 ; 28 \%-60 \%)$ for significant arrhythmia and a sensitivity of $100 \%(22 / 22 ; 95 \%$ CI $82 \%$ $100 \%)$ and specificity of $44 \%(16 / 36 ; 28 \%-62 \%)$ for serious outcome both at 90 days. The combination of BNP and high-sensitivity troponin I at 3 hours shows promise as a potential predictive marker for serious arrhythmia and serious outcome at 90 days and deserves further exploration as a rule out test to enable safe discharge of patients from the ED who are at low risk of having cardiac syncope and subsequent adverse events.
\end{abstract}

\section{Introduction}

Syncope is a common emergency department (ED) presentation but the underlying diagnosis is not apparent in $30 \%$ or more of patients after assessment and the serious adverse event rate is $7 \%$ at one month. ${ }^{1}$ Most serious outcomes are due to cardiovascular causes, which are more likely to be undetected during ED assessment. ${ }^{2}$ Previous research has aimed to identify clinical parameters that could risk stratify syncope patients presenting to the ED. ${ }^{1}$ One area of study has been assessing if cardiac biomarkers such as Brain Natriuretic Peptide (BNP) and troponin, which are known to be associated with cardiac abnormalities, could detect patients at high risk of cardiac syncope. ${ }^{3-6}$ 
BNP is a natriuretic peptide hormone, released from myocytes in association with increased pressure on the cardiac muscle wall. ${ }^{3}$ Evidence from in vivo and in vitro studies indicate that BNP release also correlates to the presence of cardiac muscle ischaemia. ${ }^{7}$ Proving this is a causal relationship is challenging, as cardiac ischaemia cannot be easily separated from other cardiac pathology known to raise BNP levels. ${ }^{8,9}$ Many cardiac diseases can lead to raised BNP through these pathophysiological mechanisms. ${ }^{3}$

Current guidelines do not recommend BNP testing for patients presenting with syncope, although there is evidence that BNP can be informative in the risk stratification of syncope patients. ${ }^{1,10-14}$ In one study, BNP levels greater than $300 \mathrm{pg} / \mathrm{mL}$ predicted $36 \%$ of serious cardiovascular outcomes and had a $99.8 \%$ negative predictive value of all-cause mortality within one month of ED presentation. ${ }^{1}$ In another study, a threshold of $40 \mathrm{pg} / \mathrm{mL}$ detected cardiac syncope with $82 \%$ sensitivity and $92 \%$ specificity. ${ }^{11}$ A specific threshold value for BNP for cardiac syncope risk has yet to be established, as highlighted by the different BNP values reported in research papers. ${ }^{1,10-13}$ Intra-person variation has also been demonstrated after syncope. ${ }^{12,13}$ In patients having an Implantable Cardiac Defibrillator (ICD) fitted or ICD batteries replaced, Ventricular fibrillation (VF) was provoked for a few seconds and BNP levels were measured..$^{13}$ In $89 \%$ of patients, BNP increased after VF and peaked at an average of 9 hours, evidence that BNP rises after a cardiac arrhythmia ${ }^{13}$ This adds a further complexity as any BNP threshold value may be dependent on blood sampling timing.

Another biomarker that has been researched for potential use in syncope patients is troponin. Troponin T and I are proteins present only in cardiac myocytes. ${ }^{5}$ They are released into blood when myocardial tissue is damaged. ${ }^{5}$ Many medical conditions and procedures can raise troponin levels, ${ }^{5}$ yet in clinical practice troponin is primarily utilised for the diagnosis of Acute Myocardial Infarction alongside clinical signs or symptoms of cardiac ischaemia. Previous studies by our group have shown that rises in troponin concentration are associated with serious outcome, including death at one month and one year after ED syncope presentation. ${ }^{6,15}$ Christ et al. identified that $40 \%$ of patients with increased troponin levels had cardiac syncope, and higher troponin in non-cardiac syncope still correlated with worse prognosis. ${ }^{16}$

A recent systematic review concluded that cardiac biomarkers may enhance accuracy of syncope risk stratification if used alongside other tests. ${ }^{14}$ This review identified much variation between studies, including different study designs, definition of Major adverse cardiac events (MACE) and differing patient populations, hence limiting conclusions. ${ }^{14}$ This current study offers a novel opportunity to analyse both BNP and troponin biomarkers in combination with arrhythmias detected on an ambulatory electrocardiogram (ECG) recorder (Zio ${ }^{\circledR}$ XT monitor). The aim of this study is to investigate if BNP and high-sensitivity troponin I predict significant arrhythmia, symptomatic significant arrhythmia and 90 dayoutcome (death and/or MACE) in unexplained ED syncope patients.

\section{Materials and Methods}

\section{Study design and setting}

This was a single-centre, prospective, cohort study. The study was conducted in the ED of the Royal Infirmary of Edinburgh (RIE): a United Kingdom tertiary centre with 120,000 adult attendances per annum. The study was granted ethical approval by the South East Scotland Research Ethical Committee (15/SS/0072). Written consent was obtained from all patients.

\section{Study population}

Patients aged 16 years or over who presented within 6 hours of an episode of syncope and whose syncope remained unexplained after ED assessment were enrolled. Exclusion criteria were obvious underlying cause after ED assessment (defined as: i) clinical history of vasovagal syncope i.e. pre-syncope symptoms and lowrisk patient according to European Society of Cardiology guidelines current at the time of study commencement; ${ }^{17}$ ii) arrhythmia on ED ECG thought to have caused syncope; iii) arrhythmia on pre-hospital ECG causing syncope; iv) pulmonary embolism diagnosed on Computed Tomography Pulmonary Angiogram (or equivalent e.g. symptoms of $\mathrm{PE}$ plus positive leg Ultrasound/Ventilation/perfusion scan/echo); v) postural hypotension (postural drop $>20 \mathrm{mmHg}$ in ED with symptoms during test and suggestive history); vi) Myocardial Infarction; ${ }^{18}$ vii) Computed Tomography brain or clinical signs/symptoms in ED showing cerebrovascular accident or subarachnoid haemorrhage; viii) evidence of haemorrhage in ED thought to have caused syncope; ix) other obvious cause of syncope apparent in ED: alcohol or illicit drugs as presumptive cause, epileptic seizure as presumptive cause (seizure activity with a $>15$ min witness reported postictal phase), stroke/transient ischemic attack as presumptive cause, preceding head trauma, hypoglycemia as presumptive cause; or $\mathrm{x}$ ) no consent available i.e. patient lacking capacity, previous recruitment into the study or patient in custody or prison.

\section{Standardized patient assessment}

Potentially eligible patients were identified by clinical staff in the ED and assessed for study inclusion by the attending clinician. If the patient fulfilled the study eligibility criteria, written consent was taken. A decision to enroll a patient was not later overturned. Patients had a Case Report Form completed in the ED, comprising demographic, historical and examination characteristics, 12-lead ECG, and radiology and standard laboratory investigations, and subsequently entered into a specially designed electronic database. ED tests not part of the study protocol were ordered at the discretion of the treating clinician, and patients were admitted, referred for outpatient investigation, or discharged according to existing ED protocols and at the discretion of the treating clinician. If a patient was admitted the reason for admission was recorded.

All enrolled patients were fitted with an ambulatory ECG monitor $\left(\mathrm{Zio}{ }^{\circledR} \mathrm{XT}\right)$. The Zio ${ }^{\circledR} \mathrm{XT}$ monitor is a lightweight, lead-wire free, single-patient-use ECG monitor that adheres to the left upper chest and records and stores up to 14 days of continuous, beat-tobeat ECG. Patients have the option of pressing a trigger button on the device and filling out a log to document symptomatic events during their wear duration, which allows for symptom-rhythm correlation in the ECG report. After a patient completes their 14-day recording, the monitor is removed from the chest and mailed to iRhythm Technologies, Inc., where the up to 14-day single-channel recording is analyzed using a combination of proprietary algorithms and review by Certified Cardiac Technicians. The findings are then reported to the ordering physician in a report that includes information on several standard arrhythmias, including atrial fibrillation and flutter, ventricular tachycardia, supraventricular tachycardia, atrioventricular pauses, heart block, atrial and ventricular ectopic beats, and other identified arrhythmias. Zio ${ }^{\circledR}$ XT monitors were placed on patients in the ED by a trained research team member, and were left on for 2 weeks.

Additionally, study participants were also given a diary in which to record any symptoms occurring during the recording period e.g. light-headedness/dizziness, syncope/presyncope, and a Zio ${ }$ XT monitor satisfaction questionnaire and contact informa- 
tion for the PATCH-ED research team in case of monitor concerns during the study period. At the end of the study period, the patient sent the monitor back to the iRhythm Clinical Centre for data processing as described and the $\mathrm{Zio}{ }^{\circledR} \mathrm{XT}$ report was then posted to a secure web site where it could then be accessed by the Edinburgh study team.

Any study participant with a serious significant arrhythmia on the Zio ${ }^{\circledR}$ XT report was contacted immediately and appropriately referred to the hospital cardiac electrophysiology service.

Serious significant arrhythmia was defined as: i) VF; ii) ventricular tachycardia (VT) $\geq 120$ beats per minute for $\geq 30$ seconds; iii) symptomatic VT; iv) third/complete degree heart block; v) symptomatic second degree heart block type II; vi) sinus pause $\geq 6$ seconds; vii) symptomatic bradycardia $<40$ beats per minute for $\geq 30$ seconds.

Patients were followed up to 90 days after presentation through hospital and primary care Electronic Patient Record (EPR) systems. Patients who had left the NHS Lothian area after one month were contacted by telephone. Serious significant arrhythmia (see above) and significant arrhythmia were defined based on Standardized Reporting Guidelines for ED Syncope Risk Stratification Research. ${ }^{19}$

Significant arrhythmia was defined as: i) non-symptomatic VT $\geq 120$ beats per minute for $<30$ seconds; ii) symptomatic sinus bradycardia $<60$ beats per minute (but $\geq 40$ beats per minute); iii) symptomatic sinus bradycardia $<40$ beats/minute for $<30$ seconds; iv) asymptomatic sinus bradycardia $<40$ beats per minute; v) sick sinus syndrome with alternating sinus bradycardia and tachycardia; vi) sinus pause $\geq 3$ seconds (but $<6$ seconds); vii) symptomatic Mobitz type I atrioventricular heart block; viii) junctional/idioventricular rhythm; ix) symptomatic supraventricular tachycardia $>100$ beats per minute; $x)$ symptomatic atrial flutter/fibrillation with ventricular rate $>100$ beats per minute; xi) symptomatic atrial flutter/fibrillation with ventricular rate $<60$ beats per minute.

Arrhythmias were also defined as symptomatic (i.e. concurrent light-headedness/dizziness, syncope/presyncope with arrhythmia) noted via triggered and diary entries, or asymptomatic. Any significant symptomatic arrhythmia was discussed with the hospital cardiac electrophysiology service. Non-recruited but potentially eligible patients were identified by a daily search of all ED EPRs to assess for potential recruitment bias.

Written patient consent included retrieval of leftover patient's baseline (admission) routine haematology (EDTA) and biochemistry (lithium heparin gel) samples from the RIE laboratory and its quantification for hs-troponin I (Abbott Architect STAT high-sensitivity troponin I assay performed in RIE laboratory) and BNP (Abbott TRIAGE point-of-care BNP test performed in the ED). ${ }^{20}$ The results of these tests were not reported to the clinician.

Additionally, one $2.7 \mathrm{~mL}$ EDTA and one $4.7 \mathrm{~mL}$ lithium heparin gel sample was taken 3 hours after admission. The lithium heparin gel sample used to measure troponin (Abbott Architect STAT highsensitivity troponin I assay performed in RIE laboratory) and the EDTA sample was used to measure BNP (Abbott TRIAGE pointof-care BNP test ${ }^{20}$ performed in the ED). The results of these tests were not reported to the clinician.

\section{Outcome measures and assessment}

The combined primary endpoint included any of the following up to 90 days after presentation: significant (including serious significant) arrhythmia, symptomatic significant (including serious significant) arrhythmia and all cause serious outcome. The latter was defined as death and/or MACE comprising myocardial infarction, ${ }^{17}$ significant arrhythmia, ${ }^{19}$ significant structural heart dis- ease,${ }^{19}$ positive electrophysiology study findings, ${ }^{19}$ permanent pacemaker or defibrillator placement, coronary artery bypass graft or coronary artery stent, cardiac valve surgery, elective cardioversion in the absence of objective evidence that tachyarrhythmia is responsible for the syncope, balloon-pump insertion, heart transplant, initiation of anti-arrhythmia medical therapy and ventricular assist device.

\section{Table 1. Patient characteristics.}

\begin{tabular}{|c|c|c|}
\hline Characteristic & & n \\
\hline Age/years & $62.8+/-19.5$ & 86 \\
\hline Male sex & $46(53)$ & 86 \\
\hline \multicolumn{3}{|l|}{ History } \\
\hline Subjective SOB & $6(7.0)$ & 86 \\
\hline Prodromal/preceding symptoms & $41(47.7)$ & 86 \\
\hline Palpitations prior to syncope & $3(3.5)$ & 86 \\
\hline Associated chest pain & $5(5.8)$ & 86 \\
\hline Associated headache & $4(4.7)$ & 86 \\
\hline Situational symptoms i.e. micturition & $6(7.0)$ & 86 \\
\hline Related to GTN use & $0(0)$ & 86 \\
\hline Related to exertion & $5(5.8)$ & 86 \\
\hline Witnessed seizure activity & $2(2.3)$ & 86 \\
\hline Estimated Length of Loss of Consciousness/mins & $1.5(1-3)^{*}$ & 58 \\
\hline \multicolumn{3}{|l|}{ Past Medical History } \\
\hline Previous episodes of syncope & $36(41.9)$ & 86 \\
\hline$>1$ episode in the last year & $17(19.8)$ & 86 \\
\hline Previous hypertension & 35 (40.7) & 86 \\
\hline Known ischemic heart disease & $11(12.8)$ & 86 \\
\hline Previous myocardial infarct & $4(4.7)$ & 86 \\
\hline Known history of valvular heart disease & $4(4.7)$ & 86 \\
\hline Previous history of cardiac arrest & $1(1.2)$ & 86 \\
\hline Known history of cardiac failure & $5(5.8)$ & 86 \\
\hline Pacemaker/Implantable defibrillator & $0(0)$ & 86 \\
\hline \multicolumn{3}{|l|}{ Examination } \\
\hline Pulse/bpm & $73.7+/-14.9$ & 86 \\
\hline Blood sugar $/ \mathrm{mmol} / \mathrm{L}$ & $6.6+/-2.1$ & 81 \\
\hline Systolic BP/mmHg & $138.6+/-27.1$ & 86 \\
\hline Diastolic BP/mmHg & $75.9+/-17.5$ & 86 \\
\hline Postural Drop ( $>20 \mathrm{mmHg}$ on standing) & $4(7.0)$ & 57 \\
\hline Systolic difference if performed & $11.1+/-10.6$ & 31 \\
\hline Saturation $\leq 94 \%$ on admission & $25(29.1)$ & 86 \\
\hline Bradycardia $\leq 50$ in ED or pre-hospital & $6(7.0)$ & 86 \\
\hline Heart murmur heard & $6(7.0)$ & 86 \\
\hline Clinical signs of heart failure present & $3(3.5)$ & 86 \\
\hline New neurological signs on examination & $0(0)$ & 86 \\
\hline FOB/Melena on PR & $0(0)$ & 10 \\
\hline \multicolumn{3}{|l|}{ Management } \\
\hline Admitted & $39(45.3)$ & 86 \\
\hline Discharged & $47(54.7)$ & 86 \\
\hline
\end{tabular}

SOB, Shortness of breath; GTN, glyceryl trinitate; BP, blood pressure; FOB, faecal occult blood; PR, per rectum.. Values are presented as mean + /- SD or n (\%) unless stated. *Median (IQR). 


\section{Sample size}

This study was a pre-planned secondary analysis of data collected as part of the PATCH-ED study, ${ }^{21}$ which aimed to recruit 100 patients to inform its primary and secondary aims.

\section{Data analysis and statistics}

Two researchers independently reviewed all clinical data and assigned endpoints with any disagreements resolved by consensus. Normally distributed variables are presented as mean $+/$ - standard deviation (SD); otherwise the median and interquartile range were used as summary measures. Mann-Whitney $\mathrm{U}$ tests were used to compare BNP at admission, 3 hours and delta change with endpoints. Receiver Operator Characteristic (ROC) curve analysis was used to assess sensitivity and specificity of BNP and hs-troponin I at different values. Although the product test threshold for the diagnosis of congestive heart failure is $100 \mathrm{pg} / \mathrm{mL}$ there are currently no standard normal values for BNP in cardiac syncope. In the absence of any standard normal values in this patient group, a threshold value of BNP was chosen with ideal clinical sensitivity and specificity. A conventional $99^{\text {th }}$ sex specific centile was chosen as the hs-troponin I threshold to be evaluated in outcome prediction analysis. Further analysis was then performed on the association between categorized BNP and hs-troponin I values and outcome which were compared using Fisher's exact test.

\section{Results}

Between 17/11/2015 and 16/06/2017, 86 patients were recruited to the study. Figure 1 details the study recruitment diagram. Baseline characteristics are shown in Table 1 and Figure 2 shows clinician's likelihood ratings of underlying cardiac syncope. Mean age was 62.8 years (range: $18.5-97.5$ years) and there were 46 males $(53 \%)$ and 40 females (47\%).

\section{0 day outcomes}

At 90 days, $24 / 86$ study participants (27\%) had a significant (including serious) arrhythmia detected by the Zio ${ }^{\circledR}$ XT monitor. There were $9 / 86$ patients $(10 \%)$ who had a symptomatic significant (including serious) arrhythmia and a serious outcome, classified as a MACE and/or death, occurred in 26/86 patients (30\%).

\section{Timing of sampling}

53 of the 86 patients had two BNP samples drawn; the mean time between sampling was 2.98 hours (range: $0.43-5.9$ hours). Two troponin samples were drawn in 45 patients with a mean time between sampling of 2.9 hours (range: 0.58-6.2 hours).

\section{Brain Natriuretic Peptide}

Median admission BNP was $23.3 \mathrm{pg} / \mathrm{mL}$ (IQR 9.0 - 90.6; $\mathrm{n}=73$ ) and median three hour BNP was $25.9 \mathrm{pg} / \mathrm{mL}$ (IQR 11.8 $102.9 ; \mathrm{n}=60$ ). Table 2 shows association between study endpoints and BNP at admission, 3 hours and delta change using ROC curve analysis. BNP at admission, three hours and change in BNP were associated with significant arrhythmia and serious outcome.

A three-hour BNP level of $20 \mathrm{pg} / \mathrm{mL}$ was chosen for further analysis and the addition of admission BNP did not improve outcome prediction enough to justify the extra clinical workload and an additional blood test for patients. 39 of 60 patients had a BNP level $>20$ $\mathrm{pg} / \mathrm{mL}$ at 3 hours which had a sensitivity of 95\% (95\% CI 74-100), specificity of 51\% (35-67), Positive Predictive Value (PPV) of 51\% (35-67) and Negative Predictive Value (NPV) of 95\% (74-100) for both significant arrhythmia and serious outcome at 90 days. This

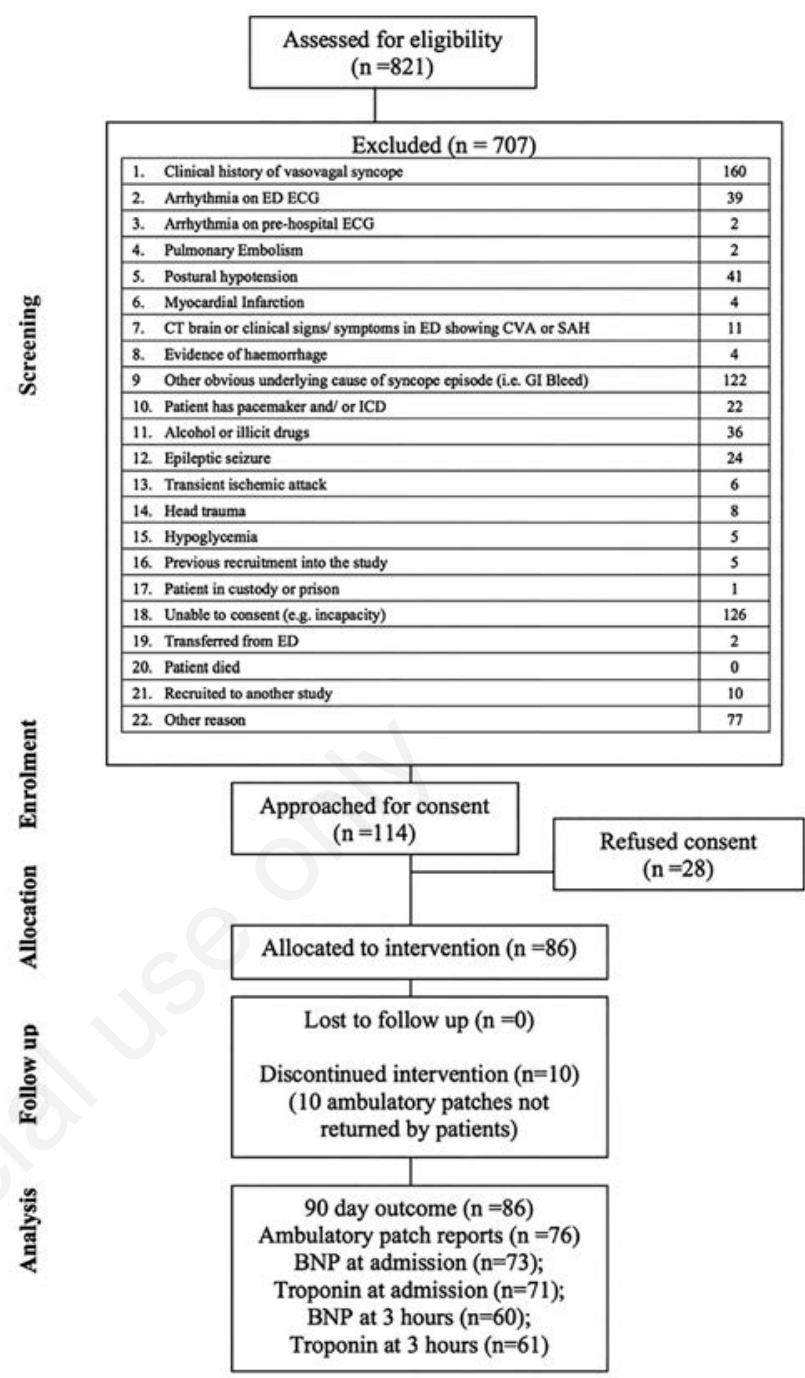

Figure 1. Study recruitment diagram. BNP, Brain Natriuretic Peptide; ED, emergency department; ECG, electrocardiogram; CVA, cerebrovascular accident; SAH, subarachnoid haemorrhage; ICD, Implantable cardiac defibrillator.

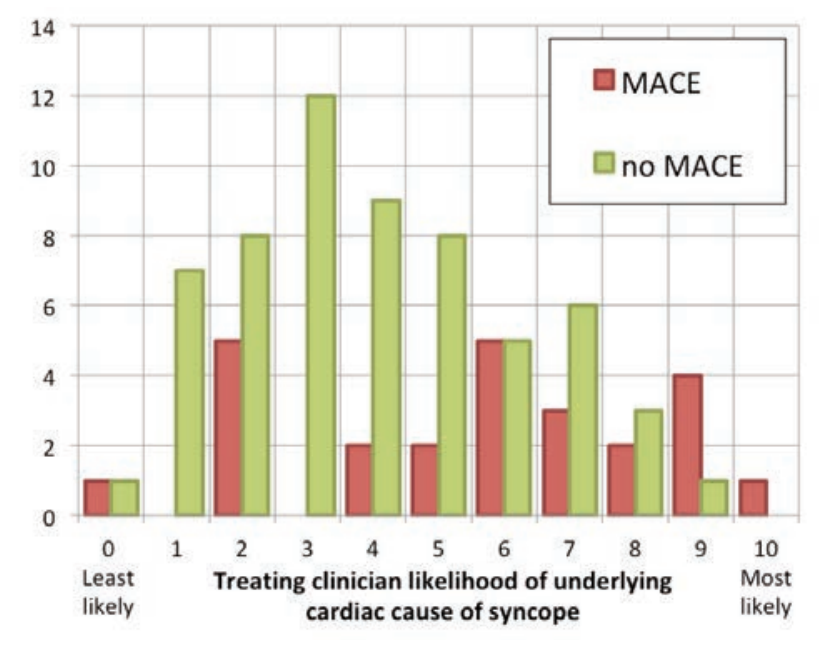

Figure 2. Frequency of treating clinician's likelihood of underlying cardiac cause of syncope rating (0 least likely-10 most likely). MACE, Major adverse cardiac events 
threshold missed an outcome in one patient, a 23 -year-old male with a BNP of $11.3 \mathrm{pg} / \mathrm{mL}$ at three hours who had a significant arrhythmia and serious outcome (non-symptomatic VT $\geq 120 \mathrm{bpm}<30$ seconds). BNP change from admission to three hours had a median difference of $3.7 \mathrm{pg} / \mathrm{mL}$ and median percentage change of $18.5 \%(\mathrm{n}=46) .7$ paired samples included a value less than the assay's lower threshold of 5 . These were resulted as $<5$ and therefore a BNP change could not be calculated. Given the test's coefficient of variation of 9.2-11.4\%, this percentage change cannot only be due to test variation. ${ }^{20}$

\section{Troponin}

Median admission hs troponin I was $3.9 \mathrm{ng} / \mathrm{L}$ (IQR 2.0-8.5; $\mathrm{n}=71$ ) and median three hour hs-troponin I was $4.9 \mathrm{ng} / \mathrm{L}$ (IQR 1.5$10.0 ; n=61) .11$ of 61 patients had a hs-troponin I above the $99 \%$ sex specific centile at three hours which was associated with significant arrhythmia and serious outcome (Table 2). Troponin on admission was significantly associated with serious outcome (Table 2). From admission to three hours, delta hs-troponin levels had median difference of $0.55 \mathrm{ng} / \mathrm{L}(\mathrm{n}=50)$ and median percentage change of $15.0 \%(n=50)$.

\section{Brain Natriuretic Peptide and Troponin}

Given that BNP and troponin at three hours were the time points most associated with significant arrhythmia and serious outcome, analysis was carried out to determine if these biomarkers in combination improved accuracy of outcome prediction (Table 3). 42 of 58 patients had a BNP $>20 \mathrm{pg} / \mathrm{mL}$ and/or high-sensitivity troponin I above the $99 \%$ sex specific centile $(\mathrm{M}>34 \mathrm{ng} / \mathrm{L}, \mathrm{F}>16 \mathrm{ng} / \mathrm{L})$ at 3 hours which had a sensitivity of $100 \%(21 / 21 ; 95 \%$ CI $81 \%$ $100 \%)$, specificity of $43 \%(16 / 37 ; 28 \%-60 \%)$, PPV of $50 \%(21 / 42$; $34 \%-66 \%)$ and NPV of $100 \%(16 / 16 ; 76 \%-100 \%)$ for significant arrhythmia at 90 days and a sensitivity of $100 \%(22 / 22 ; 95 \%$ CI $82 \%-100 \%)$, specificity of $44 \%(16 / 36 ; 28 \%-62 \%)$, PPV of $52 \%$
(22/42; 37\%-68\%) and NPV of $100 \%(16 / 16 ; 76 \%-100 \%)$ for serious outcome at 90 days.

\section{Discussion}

This study has shown that BNP is associated with adverse outcome in ED syncope patients whose underlying cause is unknown after ED assessment. High sensitive troponin alone had less value to predict adverse outcome. A combination of BNP and high sensitive troponin was able to predict all patients with subsequent significant arrhythmia and serious outcome with $100 \%$ sensitivity.

BNP at admission, 3 hours and delta change was associated with significant arrhythmia and serious outcome. BNP $>20 \mathrm{pg} / \mathrm{mL}$ alone at three hours had a NPV of $95 \%$ for significant arrhythmia and serious outcome at 90 days. This threshold of $20 \mathrm{pg} / \mathrm{mL}$ is lower than thresholds determined as predictive of cardiac syncope in other studies. ${ }^{1,11}$ However in ED clinical practice in this patient group, an important factor is that any biomarker test must be able to help the clinician safely rule out a potential adverse outcome and allow them to safely discharge the patient from the ED. High negative predictive value and sensitivity are therefore important if the test is to be used as a useful rule out test. Troponin had limited predictive value alone for the study endpoints. This finding is similar to other studies that have shown that raised troponin correlates with cardiac syncope and serious outcome, though lacks predictive power independently of other variables. ${ }^{6,16}$

Combining the biomarker tests resulted in an improved sensitivity, specificity, PPV and NPV compared to BNP alone at 3 hours. However, utilisation of both biomarkers resulted in identification of all patients who had a clinical outcome by 90 days. This study supports the recent meta-analysis that suggested using car-

Table 2. Association between study endpoints and BNP and troponin at admission, 3 hours and delta change using ROC curve analysis.

\begin{tabular}{|c|c|c|c|c|c|c|}
\hline & \multicolumn{2}{|c|}{$\begin{array}{l}\text { BNP at admission } \\
(n=71) \quad(n=60)\end{array}$} & \multicolumn{2}{|c|}{$\begin{array}{l}\text { BNP at } 3 \text { hours } \\
(n=46)\end{array}$} & \multicolumn{2}{|c|}{ Change in BNP } \\
\hline & ROC AUC & p value & ROC AUC & p value & ROC AUC & p value \\
\hline Significant arrhythmia & $0.75(0.62-0.88)$ & $0.001 *$ & $0.80(0.69-0.91)$ & $<0.0001^{*}$ & $0.78(0.63-0.93)$ & $0.001^{*}$ \\
\hline Symptomatic significant arrhythmia & $0.70(0.49-0.92)$ & 0.06 & $0.75(0.60-0.90)$ & $0.03^{*}$ & $0.76(0.64-0.91)$ & $0.03^{*}$ \\
\hline \multirow[t]{2}{*}{ Serious outcome (MACE and/or death) } & $0.77(0.65-0.89)$ & $<0.0001^{*}$ & $0.80(0.69-0.91)$ & $<0.0001^{*}$ & $0.78(0.63-0.93)$ & $0.001 *$ \\
\hline & $\begin{array}{l}\text { Troponi } \\
\text { ROC AUC }\end{array}$ & $\begin{array}{l}\text { mission } \\
\text { p value }\end{array}$ & $\begin{array}{l}\text { 71) Troponir } \\
\text { ROC AUC }\end{array}$ & $\begin{array}{l}\text { hours (n } \\
\text { p value }\end{array}$ & $\begin{array}{l}=61) \text { Change } \\
\text { ROC AUC }\end{array}$ & $\begin{array}{l}\text { onin }(n=5 \\
p \text { value }\end{array}$ \\
\hline Significant arrhythmia & $0.63(0.49-0.77)$ & 0.1 & $0.71(0.58-0.84)$ & $0.01 *$ & $0.47(0.28-0.65)$ & 0.7 \\
\hline Symptomatic significant arrhythmia & $0.44(0.28-0.59)$ & 0.6 & $0.55(0.35-0.75)$ & 0.7 & $0.37(0.16-0.58)$ & 0.3 \\
\hline Serious outcome (MACE and/or death) & $0.68(0.54-0.81)$ & $0.02 *$ & $0.74(0.61-0.86)$ & $0.003 *$ & $0.51(0.33-0.70)$ & 0.9 \\
\hline
\end{tabular}

BNP, Brain Natriuretic Peptide; ROC AUC, Receiver Operating Characteristic Area Under Curve (2 decimal places) with 95\% CI; MACE, Major adverse cardiac events. P values are ROC AUC asymptotic significance (null hypothesis; true area $=0.5$ ) and are given to 1 significant figure. *Significant at $5 \%$.

Table 3. Test characteristics for BNP at 3 hours $(>20 \mathrm{pg} / \mathrm{mL})$ or a raised troponin above the $99 \%$ sex specific centile $(\mathrm{males}>34 \mathrm{ng} / \mathrm{L}$, females $>16 \mathrm{ng} / \mathrm{L}$ ) and study outcome.

\begin{tabular}{|c|c|c|c|c|}
\hline Study Outcome $(\mathrm{n}=58+)$ & Sensitivity (\%) & Specificity (\%) & PPV (\%) & NPV (\%) \\
\hline Significant arrhythmia & $\begin{array}{c}100(95 \% \mathrm{CI} 81-100) \\
21 / 21\end{array}$ & $\begin{array}{c}43 \text { (95\%CI 28-60) } \\
16 / 37\end{array}$ & $\begin{array}{c}50 \text { (95\%CI 34-66) } \\
21 / 42\end{array}$ & $\begin{array}{c}100(95 \% \text { CI } 76-100) \\
16 / 16\end{array}$ \\
\hline Symptomatic significant arrhythmia & $\begin{array}{c}100(95 \% \mathrm{CI} 60-100) \\
8 / 8\end{array}$ & $\begin{array}{c}32(95 \% \text { CI 20-47) } \\
16 / 50\end{array}$ & $\begin{array}{c}19(95 \% \text { CI } 9-35) \\
8 / 42\end{array}$ & $\begin{array}{c}100(95 \% \text { CI } 76-100) \\
16 / 16\end{array}$ \\
\hline Serious outcome at 90 days (MACE +/- death) & $\begin{array}{c}100(95 \% \text { CI } 82-100) \\
22 / 22\end{array}$ & $\begin{array}{c}44 \text { (95\%CI 28-62) } \\
16 / 36\end{array}$ & $\begin{array}{c}52(95 \% \text { CI } 37-68) \\
20 / 42\end{array}$ & $\begin{array}{c}100 \text { (95\%CI 76-100) } \\
16 / 16\end{array}$ \\
\hline
\end{tabular}

+58 of 86 study patients had both a BNP and high sensitivity troponin drawn at 3 hours. PPV, Positive Predictive Value; NPV, Negative Predictive Value. 
diac biomarkers could aid syncope risk stratification. ${ }^{14}$

BNP and troponin both exhibited intra-patient variation between the two samples, with both increases and decreases in biomarker at three hours compared to admission baseline. For BNP this variability cannot be entirely attributed to the test's margin of error. ${ }^{20}$ Intra-patient variation does not seem to be much better than a three hour result at predicting outcome. A similar situation exists for troponin; although one participant with a 90-day arrhythmia outcome had a raised troponin at three hours only after a normal presentation troponin, further evidence for using this time point.

The mean time between samples was around three hours, although it is important to note the wide range, which could be regarded as a limitation of the study. Nevertheless, when transferring the findings into clinical practice these are representative of real world practice that is likely to occur in the ED. Our study suggests that $\mathrm{BNP}<20 \mathrm{pg} / \mathrm{mL}$ and/or high-sensitivity troponin I below the 99\% sex specific centile ( $\mathrm{M}>34 \mathrm{ng} / \mathrm{L}, \mathrm{F}>16 \mathrm{ng} / \mathrm{L})$ taken three hours after admission shows promise as a potential predictive marker for ruling out serious arrhythmia as the cause of syncope in around $30 \%$ of patients, even with some degree of variability in sample timings.

\section{Limitations of study}

There are a number of limitations to this study. It is single centre, with a small number of outcomes with no external validation. However, all patients were thoroughly investigated for arrhythmia ensuring that we can be very confident in those participants who did not have an adverse outcome, a problem befalling many syncope studies. BNP threshold was evaluated in the data set from which it was derived which will lead to optimism bias in the estimated test characteristics (i.e. sensitivity, specificity). Whist we are unaware of any possible selection effect among those patients with two marker measurements at both time intervals, compared to those with only one marker or one timing, if present, this may lead to confounding of the assessment of the predictive value of delta measurements.

\section{Application in clinical practice}

Around 780,000 to 1,300,000 patients present to EDs with syncope each year across the UK, of which around $30 \%$ do not have a cause identified after ED assessment. Utilising this biomarker strategy could potentially inform clinical management of patients with unexplained syncope and deserves further evaluation in an external validation study. One clinical strategy that this study might support is an initial combined biomarker test at 3 hours post ED attendance, with outpatient ambulatory monitoring for those with a raised biomarker, and safe discharge from the ED for those patients with negative biomarkers who are at low risk of having cardiac syncope and subsequent adverse events.

\section{Conclusions}

Syncope is a common medical presentation to the ED, which may be due to underlying arrhythmia. Challenges remain in differentiating between patients with cardiac syncope and low risk syncope causes. Previous studies have demonstrated that cardiac biomarkers can assist syncope risk stratification. In this sample all patients with a serious outcome (MACE or death) and significant arrhythmia within 90 days of ED presentation were predicted by $\mathrm{BNP}>20 \mathrm{pg} / \mathrm{mL}$ or high-sensitivity troponin I above the $99^{\text {th }}$ centile taken at three hours post ED admission. This tool deserves further exploration as a rule out test to enable safe discharge of patients from the ED who are at low risk of having cardiac syncope and subsequent adverse events.

\section{References}

1. Reed MJ, Newby DE, Coull AJ, et al. Risk stratification of syncope in the emergency department: The ROSE study. J Am Coll Cardiol 2010;55:713-21.

2. Reed MJ, Henderson SS, Newby DE, Gray AJ. One-year prognosis after syncope and the failure of the ROSE decision instrument to predict 1 year adverse events. Ann Emerg Med 2011;58:250-6.

3. Hall C. Essential biochemistry and physiology of (NT-pro)BNP. Eur J Heart Fail 2004;6: 257-60.

4. Chronic heart failure-NICE Pathways; 2017. Available from: http://pathways.nice.org.uk/pathways/chronic-heart-failure. Accessed: 1 September 2017.

5. Korff S, Katus H, Giannitsis E. Differential diagnosis of elevated troponins. Heart 2006; 92:987-93.

6. Reed M, Mills N, Weir C. Sensitive troponin assay predicts outcome in syncope. Emerg Med J 2012;29:1001-3.

7. Yeo K, Lee H, Wong K, Foote R. Can exercise-induced changes in b-type natriuretic peptides be used to detect cardiac ischemia? J Cardiac Fail 2005;11:S59-S64.

8. Arjamaa O, Nikinmaa M. Does hypoxia directly regulate the natriuretic peptide system? Int J Cardiol 2012;154:372.

9. Costantino G, Barbic F, Solbiati M, et al. The elusive mechanisms of B-type natriuretic peptides rise after ventricular fibrillation. Int J Cardiol 2012;156:247-8.

10. Reed M, Newby D, Coull A, et al. Role of brain natriuretic peptide $(\mathrm{BNP})$ in risk stratification of adult syncope. Emerg Med J 2007;24:769-73.

11. Tanimoto K, Yukiiri K, Mizushige K, et al. Usefulness of brain natriuretic peptide as a marker for separating cardiac and noncardiac causes of syncope. Am J Cardiol 2004;93:228-30.

12. Reed M, Gibson L. The effect of syncope on brain natriuretic peptide. Emerg Med J 2011;28:1066-7.

13. Costantino G, Solbiati M, Sagone A, et al. Time course of B-type natriuretic peptides changes after ventricular fibrillation: Relationships with cardiac syncope. Int J Cardiol 2011;153: 333 5.

14. Thiruganasambandamoorthy V, Ramaekers R, Rahman M, S et al. Prognostic value of cardiac biomarkers in the risk stratification of syncope: a systematic review. Int Emerg Med 2015;10:1003-14.

15. O'Neill A, Reed MJ. Troponin use in emergency department syncope. Eur J Emerg Med 2016;23:77-8.

16. Christ M, Geier F, Popp S, et al. Diagnostic and prognostic value of high-sensitivity cardiac troponin $\mathrm{t}$ in patients with syncope. Am J Med 2015;128:161-70.e1.

17. Guidelines for the diagnosis and management of syncope (version 2009). The Task Force for the Diagnosis and Management of Syncope of the European Society of Cardiology (ESC). Eur Heart J 2009;30:2631-71.

18. Thygesen K, Alpert JS, Jaffe AS, et al. Third universal definition of myocardial infarction. Eur Heart J 2012;33:2551-67.

19. Sun BC, Thiruganasambandamoorthy V, Dela Cruz J, et al. Standardized reporting guidelines for emergency department syncope risk stratification research. Acad Emerg Med 2012;19:694702.

20. Triage ${ }^{\circledR}$ BNP Test Product Insert. San Diego, CA: Alere; 2015.

21. Reed MJ, Grubb NJ, Lang CC, et al. Diagnostic yield of an ambulatory patch monitor in patients with unexplained syncope after initial evaluation in the Emergency Department: The PATCH-ED study. Emerg Med J 2018;0:1.9. 